\title{
Magnetohydrodynamic simulations of the collapsar model for early and late evolution of gamma-ray bursts
}

\begin{abstract}
By DANIEl Proga
Physics Department, University of Nevada, Las Vegas, Nevada 89154, USA

I present results from magnetohydrodynamic (MHD) simulations of a gaseous envelope collapsing onto a black hole. These results support the notion that the collapsar model is one of most promising scenarios to explain the huge release of energy in a matter of seconds associated with Gamma Ray Bursts (GRB). Additionally, the MHD simulations show that at late times, when the mass supply rate is expected to decrease, the region in the vicinity of the black hole can play an important role in determining the rate of accretion, its time behaviour, and ultimately the energy output. In particular, the magnetic flux accumulated around the black hole can repeatedly stop and then restart the energy release. As proposed by Proga and Zhang, the episode or episodes of reoccurring of accretion processes can correspond to X-ray flares discovered recently in a number of GRBs.
\end{abstract}

Keywords: accretion, accretion discs - MHD - methods: numerical gamma-rays: bursts

\section{Introduction}

The collapsar model proposes that long duration gamma-ray bursts (GRBs) are powered by accretion onto the collapsed iron core of a massive star (Woosley 1993 and Paczyński 1998). As gas accretes at a very high rate $\left(\sim 1 \mathrm{M}_{\odot} \mathrm{s}^{-1}\right)$, large neutrino and magnetic fluxes, a powerful outflow, and a GRB are produced. This model is strongly supported by the association of long-duration GRBs with stellar collapse (e.g., Hjorth et al. 2003; Stanek et al. 2003) and by theoretical studies of stellar collapse (MacFadyen \& Woosley 1999; Popham, Woosley \& Fryer 1999; Proga et al. 2003; Mizuno et al. 2004; Fujimoto et al. 2006).

Recent GRB observations obtained with Swift provide new challenges to the model as early X-ray afterglow lightcurves of nearly half of the long-duration GRBs show X-ray flares (Burrows et al. 2005; Romano et al. 2006; Falcone et al. 2006). The model must then account for an initial powerful burst of energy and for an extended activity in a form of X-ray flares.

\section{MHD simulations}

I present results from direction simulations of early evolution of stellar collapse and explore the implications of these and other simulations to infer the physical 
conditions in the vicinity of a black hole $(\mathrm{BH})$ during the late phase of evolution, i.e., when most of the stellar mass is accreted. To study the extended GRB activity, one would need to follow the collapse of the entire star. However, such studies are beyond current computer and model limits.

\section{(a) Early time evolution}

Proga et al. (2003) performed the first MHD simulation of a collapsar model. Their simulation begins after the $1.7 \mathrm{M}_{\odot}$ iron core of a $25 \mathrm{M}_{\odot}$ presupernova star has collapsed and follows the ensuing accretion of the $7 \mathrm{M}_{\odot}$ helium envelope onto the central $\mathrm{BH}$ formed by the collapsed iron core. A spherically symmetric progenitor model is assumed, but the symmetry is broken by the introduction of a small, latitude-dependent angular momentum and a weak split-monopole magnetic field. The simulation includes pseudo-Newtonian potential, a realistic equation of state, photodisintegration of bound nuclei and cooling due to neutrino emission.

The simulation shows that the early phase of evolution starts with a transient episode of infall. Then the rotating gas starts to pile up outside the $\mathrm{BH}$ and forms a thick accretion torus bounded by a centrifugal barrier near the rotation axis. Soon after the torus forms (i.e., within a couple of orbital times at the inner edge), the magnetic field is amplified by the magnetorotational instability (MRI, e.g., Balbus \& Hawley 1998) and shear. MRI facilitates outward transport of angular momentum in the torus and enables torus accretion onto a BH. Another important effect of magnetic fields is that the torus produces a magnetized corona and an outflow. The presence of the corona and outflow is essential to the evolution of the inner flow at all times and to the entire flow close to the rotational axis during the latter phase of the evolution. The outflow very quickly becomes sufficiently strong to overcome supersonically infalling gas and to escape the star. The top left panel in Fig. 1 shows a schematic picture on the inner most part of the flow. The main conclusion from the simulation is that, within the collapsar model, MHD effects alone are able to launch, accelerate and sustain a strong polar outflow.

Other important insights gained from the MHD simulation include: 1) In the accretion torus, the toroidal field dominates over the poloidal field and the gradient of the former drives a polar outflow; 2) The polar outflow can be Poynting fluxdominated; 3) The polarity of the toroidal field can change with time; 4) The polar outflow reaches the outer boundary of the computational domain $\left(5 \times 10^{8} \mathrm{~cm}\right)$ with an expansion velocity of $0.2 \mathrm{c}$; 5) The polar outflow is in a form of a relatively narrow jet (when the jet breaks through the outer boundary its half opening angle is $\left.5^{\circ}\right)$; 6) Most of the energy released during the accretion is in neutrinos, $L_{\nu}=$ $2 \times 10^{52} \mathrm{erg} \mathrm{s}^{-1}$. Neutrino driving will increase the outflow energy (e.g., Fryer \& Mészáros 2003 and references therein), but could also increase the mass loading of the outflow if the energy is deposited in the torus.

Due to limited computing time, Proga et al.'s (2003) simulation was stopped at $t=0.28215 \mathrm{~s}$, which corresponds to 6705 orbits of the flow near the inner boundary. The total mass and angular momentum accreted onto the BH during the simulation are $0.1 \mathrm{M}_{\odot}$ and $3 \times 10^{39} \mathrm{~g} \mathrm{~cm}^{2} \mathrm{~s}^{-1}$, respectively. Thus, the simulation captured just the beginning of the accretion and one expects the accretion to continue much longer, roughly the collapse timescale of the Helium core $(\sim 10 \mathrm{~s})$. However, one 
also expects a decrease of the mass supply rate with time, especially in the late phase of activity, because the stellar mass density decreases with increasing radius.

The long time evolution of MHD accretion flows was studied by Proga \& Begelman (2003) who explored simulations very similar to the one in Proga et al. (2003) but with much simpler physics (i.e., an adiabatic equation of state, no neutrino cooling or photodisintegration of helium). Next, I present some key results from Proga \& Begelman (2003) as they are relevant to late evolution of a collapsing star.

\section{(b) Late time evolution}

The early phase of the time evolution and the dynamics of the innermost flow, are very similar in simulations present in Proga et al. (2003) and Proga \& Begelman (2003). In particular, after an initial transient behaviour, the flow settles into a complex convolution of several distinct, time-dependent flow components including an accretion torus, its corona and outflow, and an inflow and outflow in the polar funnel (see the bottom left panel in Fig. 2).

However, the late evolution of Proga \& Begelman's (2003) simulations shows that the torus accretion can be interrupted by a strong poloidal magnetic field in the vicinity of a $\mathrm{BH}$ (compare the bottom left and right panels in Fig. 2). Thus the region in the vicinity of the $\mathrm{BH}$ and the $\mathrm{BH}$ itself can play an important role in determining the rate and time behaviour of the accretion and the energy output.

Motivated by this result, Proga \& Zhang (2006) conjectured that the energy release can be repeatedly stopped and then restarted, provided the mass supply rate decreases with time even if the decrease is smooth (see Fig. 1). Proga \& Zhang appealed to the fact that, as mass is being accreted onto a BH, the magnetic flux is accumulating in the vicinity of the BH. Eventually, this magnetic flux must become dynamically important and affect the inner accretion flow, unless the magnetic field is very rapidly diffused. Analytic estimates derived by Proga \& Zhang show that the model can account for the observed features of the X-ray flares.

\section{Conclusions}

Both numerical and theoretical models of magnetized accretion flows support the notion that long duration GRBs can be explained by the collapsar model. The models also show that the innermost part of the flow and accretor can respond actively to changes of the accretion flow at larger radii. In particular, the innermost accretion flow can be halted for a very long time as to account for an extended activity of GRBs with X-ray flares.

I acknowledge support from NASA under ATP grant NNG06GA80G

\section{References}

Balbus, S. A., \& Hawley, J. F. 1998 Instability, turbulence, and enhanced transport in accretion disks, Rev. Mod. Phys., 70, 1-53

Burrows, D. N. et al. 2005 Bright X-ray flares in gamma-ray burst afterglows. Science, 309, 1833-1835

Article submitted to Royal Society 
Falcone, A. et al. 2006 the giant X-Ray flare of GRB 050502B: Evidence for late-time internal engine activity. ApJ, 641, 1010-1017

Fryer, C. L., \& Mészáros, P. 2003 Neutrino-driven explosions in gamma-ray bursts and hypernovae. ApJ, 588, L25-L28

Fujimoto, S., et al. 2006 Magnetohydrodynamic simulations of a rotating massive star collapsing to a black hole. ApJ, 644, 1040-1055

Hjorth, J., et al. 2003 A very energetic supernova associated with the gamma-ray burst of 29 March 2003. Nature, 423, 847-850

MacFadyen, A., \& Woosley, S.E. 1999 Collapsars: gamma-ray bursts and explosions in "failed supernovae". ApJ, 524, 262-289

Mizuno, Y., Yamada, S., Koide, S., \& Shibata, K. 2004 General relativistic magnetohydrodynamic simulations of collapsars. ApJ, 606, 395-412

Paczyński, B. 1998 Are gamma-ray bursts in star-forming regions? ApJ, 494, L45-L48

Popham, R., Woosley, S.E., \& Fryer, C. 1999 Hyperaccreting black holes and gamma-ray bursts. ApJ, 518, 356-374

Proga, D., \& Begelman, M.C. 2003 Accretion of low angular momentum material onto black holes: two-dimensional magnetohydrodynamic case. ApJ, 592, 767-781

Proga, D., MacFadyen, A. I., Armitage, P. J., \& Begelman, M. C. 2003 Axisymmetric magnetohydrodynamic simulations of the collapsar model for gamma-ray bursts. ApJ, 599, L5-L8

Proga D., Zhang, B. 2006 The late time evolution of gamma-ray bursts: ending hyperaccretion and producing flares. MNRAS, 370, L61-L65

Romano, P. et al. 2006 X-ray flare in XRF 050406: evidence for prolonged engine activity. A\&A, 450, 59-68

Stanek, K.Z., et al. 2003 Spectroscopic discovery of the supernova 2003dh associated with GRB 030329. ApJ, 591, L17-L20

Woosley, S.E. 1993 Gamma-ray bursts from stellar mass accretion disks around black holes. ApJ, 405, 273-277 

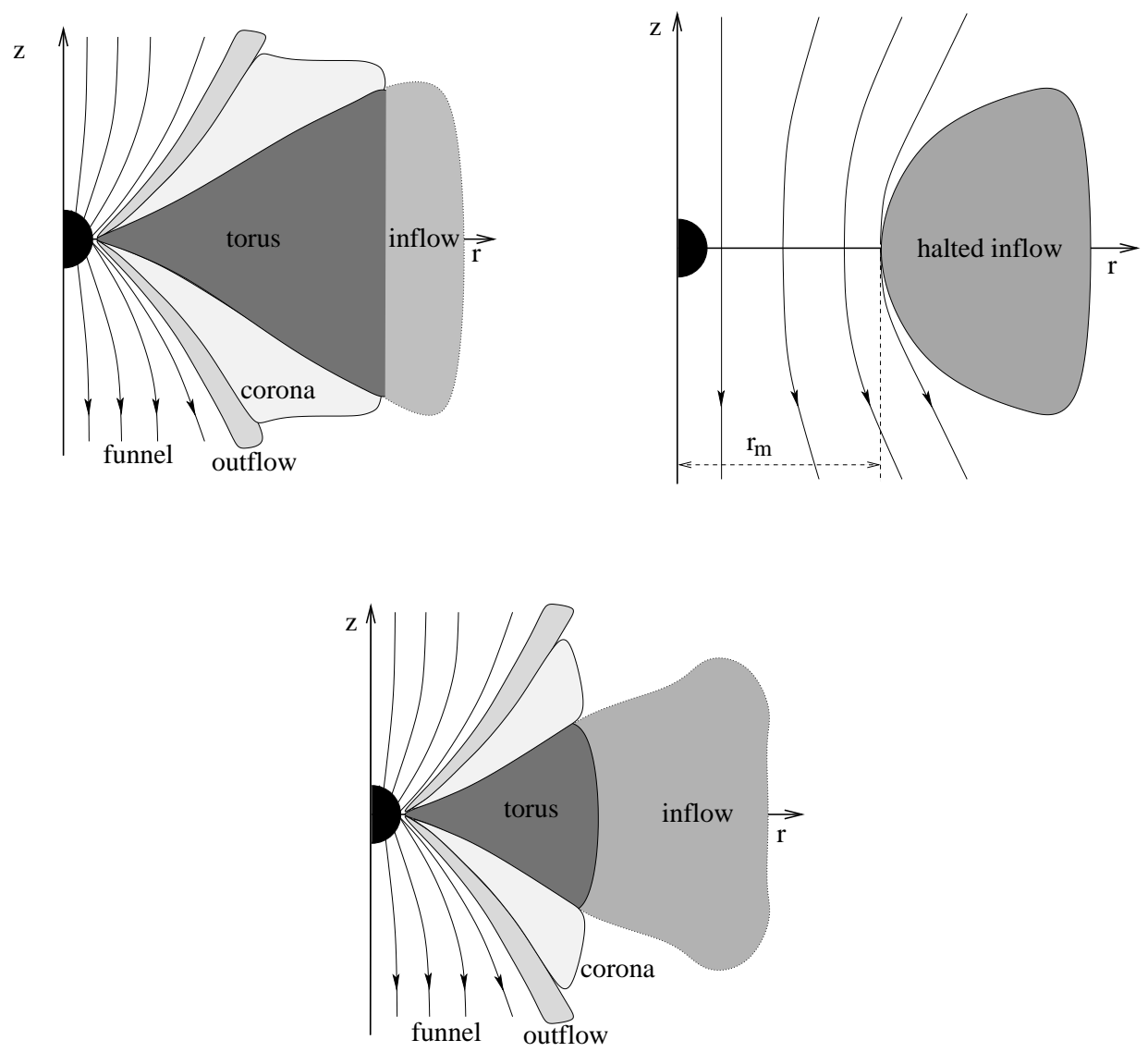

Figure 1. General structural features of the inner MHD flow during three different accretion stages: (1) The inner flow during the hyperaccretion (left top panel) when a powerful jet forms and a strong poloidal magnetic field is being accumulated at the center. The hyperaccretion can not be sustained because the mass supply rate from the outer inflow drops with time (hence different shades used for the torus and inflow). (2) The halted inflow (right top panel) when the hyperaccretion ended and the inflow rate is relatively low. During this stage the magnetic field accumulated earlier can support the gas against gravity. Consequently the inflow almost stops at the distance comparable to the magnetospheric radius, $r_{m}$. This stage ends when the surface density of the flow is too high for the magnetic field to support the gas. (3) The inner flow when the magnetosphere is squashed by the gas accumulated in the front of the inflow (bottom panel). The accretion torus is rebuilt and a powerful jet is regenerated. The accretion rate at this stage is lower than the hyperaccretion rate but higher than the inflow rate. During the late accretion the inner flow switches between the second and third stage and the third stage corresponds to the time when X-ray flares are produced. This cartoon illustrates the situation when the central magnetic flux is conserved (i.e., the solid lines with arrows correspond to the magnetic field lines at the center; for the clarity of the cartoon, the magnetic field lines of other flow components are not drawn.) 

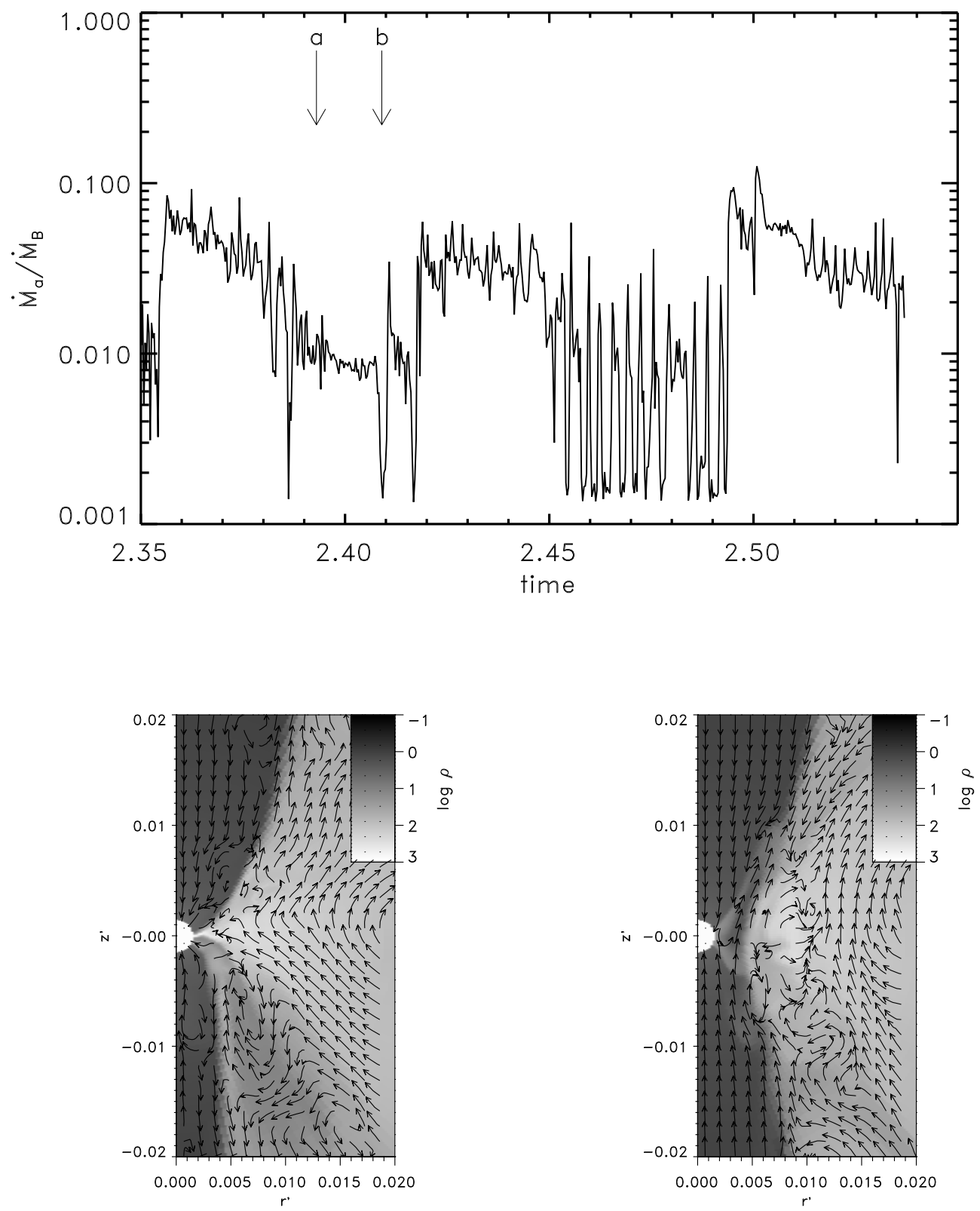

Figure 2. Top Panel: Late time evolution of the mass accretion rate in units of the Bondi rate, for Proga \& Begelman's (2003) run D. Vertical arrows mark times corresponding to the leff and right snapshots shown in the bottom row of panels (arrow a and b, respectively). Bottom Row of Panels: Maps of logarithmic density overplotted by the direction of the poloidal velocity. These panels compare the inner flow in two different accretion states mark by arrows in the top panel (see also fig. 1). The time in the top panel is in units of the Keplerian orbital time at the Bondi radius, $R_{B}$. The length scale and density in the bottom panels is in the units of $R_{B}$ and the density at infinity, $\rho_{\infty}$ respectively. In this simulation, the ratio between the Bondi radius and $\mathrm{BH}$ radius is 1200 . 